\section{Comparative Biological Effectiveness of the Gibberellins}

SINCE the isolation ${ }^{1}$ of gibberellin $A$ (later identified ${ }^{2}$ as a mixture of gibberellins $A_{1}, A_{2}$ and $A_{3}$ ), and more recently the discovery ${ }^{8}$ of gibberellin $A_{4}$, numerous physiological responses have been induced in plants ${ }^{4}$ with the gibberellins. Takahashi et al. ${ }^{8}$ reported that $A_{1}, A_{2}$ and $A_{3}$ all possessed strong physiological activities in stimulating the elongation of rice seedlings. Apart from this reference, the gibberellins have not been evaluated in terms of specific biological activities. The comparative effectiveness of gibberellins $A_{1}, A_{2}, A_{3}$ and $A_{4}$ and the methyl esters of $A_{1}$ and $A_{3}$ in promoting vegetative extension, flowering of facultative long-day annuals and in fruit setting is now reported ${ }^{5}$.

Beans (Phaseolus vulgaris, var. Blue Lake) were germinated in quartz sand, transferred to solution cultures, and after $24 \mathrm{hr}$. $10 \mu \mathrm{l}$. of a $3 \times 10^{-3} \mathrm{M}$ solution of a gibberellin or derivative was applied to the stem apex. Epicotyl elongation was determined after $48 \mathrm{hr}$. Gibberellins $A_{1}, A_{2}$ and $A_{3}$, applied to the stem apex, resulted in significantly greater epicotyl extension than the methyl esters of $A_{1}$ and $A_{3}$ (Table 1). No significant differences were apparent among the gibberellins. All produced significantly longer epicotyls than the controls. By contrast, in unpublished studies (with B. K. Gaur), gibberellins $A_{1}$ and $A_{3}$ applied to one of the primary leaves of the bean produced significantly longer epicotyls than $A_{2}$. This has suggested a limitation in absorptiontransport of gibberellin $A_{2}$.

Table 1. Comparative Biologioal Activity of GibBerdilins and

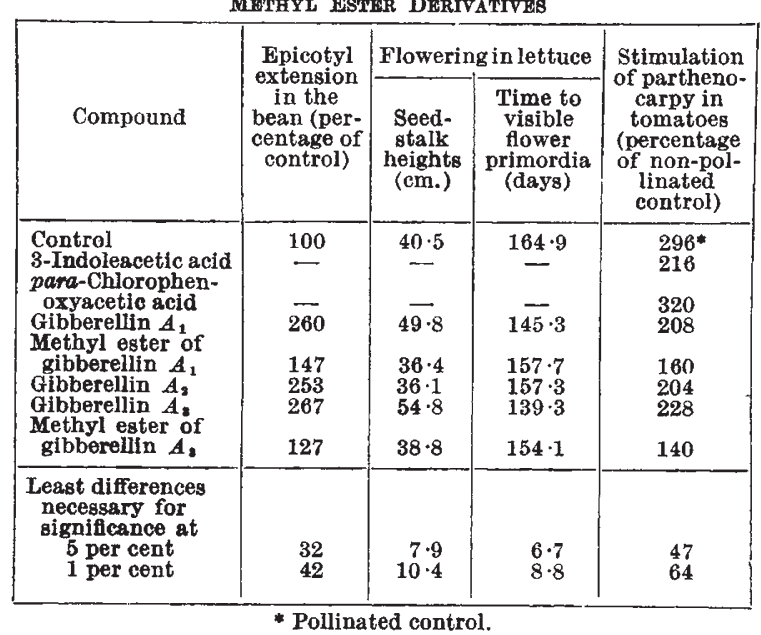

The effects of the gibberellins and derivatives on flowering were determined with lettuce (Lactuca sativa, var. Great Lakes) and dill (Anethum graveolens) grown in pot cultures and treated initially at the 6-7 leaf stage with $10 \mu \mathrm{l}$. of the solution described above. Treatments were repeated after two and four weeks. All plants were grown at a night temperature of $18^{\circ} \mathrm{C}$. and at the prevailing winter photoperiod $(9-11 \mathrm{hr}$.$) . The time required for flower primordia$ to appear in lettuce was significantly reduced and seedstalk heights were increased by treatment with gibberellins $A_{1}$ and $A_{3}$. Plants treated with $A_{2}$ or the methyl esters of $A_{1}$ and $A_{3}$ flowered slightly earlier than controls, but seedstalk elongation was not affected. Furthermore, no heads formed on plants following treatment with $A_{1}$ or $A_{3}$, whereas bolting followed heading on plants treated with $A_{2}$, the methyl esters of $A_{1}$ and $A_{3}$, and the controls. Similar effects on acceleration of flowering and seedstalk heights were observed with dill.

Comparative fruit-setting activities, in the absence of pollination, were determined with the tomato (Lycopersicon esculentum, var. Michigan-Ohio Hybrid). Three flower buds from the first cluster were emas. culated approximately $24 \mathrm{hr}$. before anthesis. $10 \mu \mathrm{l}$. of a $3 \times 10^{-3} M$ solution of the gibberellins, methyl esters of $A_{1}$ and $A_{3}$, indoleacetic acid or para-chlorophenoxyacetic acid were then applied to the ovary, and its diameter measured after six days. Solutions of gibberellins $A_{1}, A_{2}$ and $A_{3}$ and indoleacetic acid were equally effective in stimulating growth of ovaries, but less so than $p$-chlorophenoxyacetic acid at $3 \times$ $10^{-8} M$ concentration. At a lower concentration $(3 \times$ $\left.10^{-5} M\right)$, however, $A_{1}, A_{2}$ and $A_{3}$ were as effective as $p$-chlorophenoxyacetic acid and more so than indoleacetic acid. As in other responses the methyl esters of $A_{1}$ and $A_{3}$ were less active than their free acids.

Preliminary studies have indicated that gibberellin $A_{4}$ was equal in activity to $A_{1}$ for promotion of epicotyl extension of the bean and parthenocarpy in tomatoes. It was, however, less effective in stimulating stem elongation and flowering in lettuce and dill.

A consideration of the comparative biological activities of the gibberellins relative to epicotyl extension in beans, stem elongation and flowering in lettuce and dill, and parthenocarpic fruit growth in tomatoes has established the following order of activity : $A_{3}>A_{1} \simeq A_{4}>A_{2}>$ methyl ester $A_{1} \simeq$ methyl ester $A_{3}$.

We thank Dr. Y. Sumiki, University of Tokyo, for gibberellins $A_{1}, A_{2}$ and $A_{4}$ and Dr. J. F. Grove, Imperial Chemical Industries, Ltd., for $A_{3}$ and the methyl esters. These studies were supported in part by grants from Chas. Pfizer and Co., Inc., and Merck and Co., Inc.

\section{J. Bukovac}

S. H. WITTWER

Department of Horticulture,

Michigan State University, East Lansing, Michigan. March 19. ${ }^{2}$ Yabuta, T., and Sumiki, Y., J. Agric. Chem. Soc. Japan, 14, 1526

${ }^{2}$ Takahashi, N., Kitamura, H., Kawarada, A., Seta, Y., Takai, M. Tamura, S., and Sumiki, Y., Bull. Agric. Chem. Soc., Japan, 18, 267 (1955). 3 Takahashi, N., Seta, Y. Kitamura, H., and Sumiki, Y., Bull. Agric.
Chem. Soc. Japan, 21 , 396 (1957).

Stowe, B. B., and Yamaki, T., "Ann. Rev. Plant Physiol.", 8, 181 (1957).

5 Journal Article No. 2231 from the Michigan Agricultural Experiment Station.

\section{Effects of Alkoxyglycerols and especially Selachyl Alcohol on the Bone Marrow in connexion with Irradiation Treatment and in Leukzemia Therapy}

IN connexion with irradiation treatment it has been shown that alkoxyglycerols to a certain extent prevent and cure leuko- and thrombo-cytopmia'. It was found that the effect was related to the amount of alkoxyglycerols administered per os, Above a certain dosage there is less effect. 